\title{
A133 BREAKAGE OF B CELL TOLERANCE BY ANTIGENS ON FOLLICULAR DENDRITIC CELLS
}

Mohey Eldin El Shikh, ${ }^{1}$ Rania M El Sayed, ${ }^{1}$ Maciej Kmieciak, ${ }^{2,3}$ Masoud Manjili, ${ }^{2,3}$ Andras Szakal, ${ }^{4}$ Costantino Pitzalis, ${ }^{1}$ John Tew ${ }^{2}{ }^{1}$ Experimental Medicine \& Rheumatology, William Harvey Research Institute, Barts and The London School of Medicine, Queen Mary University of London, London, UK; ${ }^{2}$ Microbiology and Immunology; ${ }^{3}$ Massey Cancer Centre; ${ }^{4}$ Anatomy and Neurobiology, VCU School of Medicine, Virginia Commonwealth University, Richmond, Virginia, USA

10.1136/ard.2010.148999.9

Background and objectives Autoimmune disorders frequently display autoreactive germinal centres (GCs) with immune complex (IC)-bearing follicular dendritic cells (FDCs) suggesting that FDCs are involved in the pathogenesis of autoimmune diseases. The authors recently described induction of $\mathrm{T}$ cell-independent (TI) antibody (Ab) responses to $\mathrm{T}$ dependent (TD) antigens (Ags) by presenting the TD Ags on FDCs. Rather than presenting peptide fragments, FDCs multimerize monomeric Ags by trapping them in ICs via FDC-Fc $\gamma$ RIIB and then present them polyvalently with a characteristic periodic spacing between epitopes of 200-500 . This allows extensive $B$ cell receptor (BCR) cross-linking that induces TI $B$ cell activation, GC formation, and Ag-specific Ig secretion in less than $48 \mathrm{~h}$ in the presence of FDC-derived co-stimulatory signals. These correlations prompted the hypothesis that multimerized self/neo-Ags on FDCs would break B cell tolerance; induce autoreactive GC formation, and auto-Ab responses.

Materials and methods ICs of autologous tumour necrosis factor $\alpha$ or $\operatorname{IgE}$, were injected into BALB/c mice. In addition, soluble hen egg lysozyme transgenic (sHEL-Tg) and rat neu-Tg FVBN202 mice were challenged with HEL or the subdomain II of the extracellular domain of rat neu (ECDII), respectively, in the form of ICs. These auto/neo Ags were selected because Abs reactive with these Ags are used clinically or, in the case of sHEL-Tg mice, the profound tolerance to sHEL has been well verified.

Results Remarkably, in all cases, the ICs were trapped by FDCs; and autoreactive GCs, plasmablast differentiation and strong auto-Ab responses were induced within $48 \mathrm{~h}$. In marked contrast, free $\mathrm{Ag}$ that would have unfettered access to BCRs did not load on FDCs and did not induce detectable auto-Abs. Conclusions This is the first report of deliberately inducing auto-Abs by self/neo-Ags on FDCs. The data provide a mechanism by which FDCs may contribute to autoimmunity, and suggest a novel approach for targeting mediators of chronic inflammation, hypersensitivity and cancer. Therapeutic induction of auto-Abs by FDC-ICs may provide self-regulated high affinity Abs efficient in long-term disease control. 